\title{
The mechanism of phenytoin crystal growth
}

\author{
Gail L. Zipp ${ }^{1}$ and Naír Rodríguez-Hornedo \\ College of Pharmacy, The University of Michigan, Ann Arbor, MI 48109-1065 (USA)
}

(Received 6 August 1992)

(Modified version received 8 March 1993)

(Accepted 18 March 1993)

Key words: Phenytoin; Crystal growth; Kinetics; Mechanism

\section{Summary}

Phenytoin crystal growth kinetics have been measured as a function of $\mathrm{pH}$, supersaturation and temperature in phosphate buffer. Incorporation of growth units into the crystal lattice was not influenced by diffusion from the bulk to the solid surface. Thus, the rate limiting step for phenytoin growth is surface integration. Phenytoin crystal growth is via a screw-dislocation mechanism; this mechanism explains the observed dependence of growth rate on supersaturation and the increase in growth rate with $\mathrm{pH}$.

\section{Introduction}

An understanding of crystallization mechanisms is necessary for the prediction of solids properties which influence the dissolution rate and bioavailability of oral dosage forms such as particle size, size distribution and morphology. In addition, formulation of non-precipitating dosage forms requires an understanding of the conditions under which crystals will form so that limiting conditions for non-precipitating systems can be evaluated.

Many systems of pharmaceutical importance contain weak acids or weak bases. Since the solu-

Correspondence to: N. Rodríguez-Hornedo, College of Pharmacy, The University of Michigan, Ann Arbor, MI 48109-1065, U.S.A.

1 Present address: The Upjohn Company, 301 Henrietta St, Kalamazoo, MI 49007, U.S.A. bility of these compounds is dependent on $\mathrm{pH}$, in vitro and in vivo crystallization of the free acid or base can occur as a result of a $\mathrm{pH}$ change. In vivo, precipitation follows either dissolution of a solid phase or dilution of a solution of the salt of a weak acid or base. Precipitation via a $\mathrm{pH}$ change is also commonly used in industrial crystallization.

Previous investigations have highlighted this phenomenon. Serajuddin (1990) studied the dissolution of the sodium salt of phenytoin (NaDPH) and observed crystallization of the acid (DPH) in the $\mathrm{pH}$ range of 1-8. Higuchi et al. (1965) observed a similar behavior during the dissolution of sodium tolazamide. Newton and Kluza (1980) and Salem et al. (1980) have reported crystallization of DPH as a result of dilution of a solution of NaDPH. Even though the more soluble salts are often the choice in a formulation, crystallization of the unionized species may be a controlling factor in drug delivery. 
The purpose of the work presented here was to study the mechanism and kinetics of DPH crystal growth as a function of supersaturation, $\mathrm{pH}$ and temperature.

\section{Background}

Phenytoin (Mol. Wt 252.3) is a weak acid whose structure is shown in Fig. 1. The sodium salt is highly dissociated in water and the anion can react to form the undissociated acid according to the following equilibria:

$$
\begin{aligned}
& \mathrm{Na}^{+}(\mathrm{DPH})^{-} \stackrel{\mathrm{H}_{2} \mathrm{O}}{\rightleftharpoons} \mathrm{Na}^{+}+(\mathrm{DPH})^{-} \\
& (\mathrm{DPH})^{-}+\mathrm{H}_{3} \mathrm{O}^{+} \rightleftharpoons \mathrm{DPH}+\mathrm{H}_{2} \mathrm{O}
\end{aligned}
$$

The total phenytoin solubility, $S$, can be expressed as a function of the hydrogen ion concentration, $\left[\mathrm{H}^{+}\right]$, the solubility of the unionized form, $S_{0}$, and the dissociation constant for DPH, $K_{\mathrm{a}}$ :

$$
S=S_{0}+(\mathrm{DPH})^{-}=S_{0}+K_{\mathrm{a}} S_{0} /\left[\mathrm{H}^{+}\right]
$$

This can be transformed into the more common $\mathrm{pH}$ and $\mathrm{p} K_{\mathrm{a}}$ notation as:

$\log \left(S / S_{0}-1\right)=\mathrm{pH}-\mathrm{p} K_{\mathrm{a}}$

Schwartz et al. (1973) measured the solubility of the free acid in phosphate buffer at an ionic

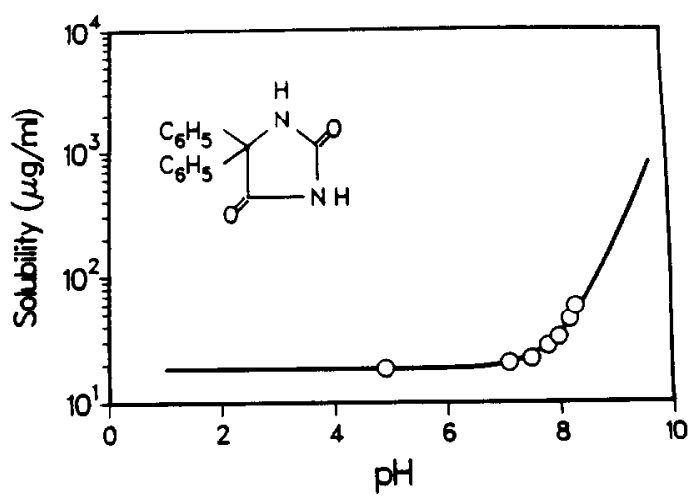

Fig. 1. Phenytoin solubility as a function of $\mathrm{pH}$ at $25^{\circ} \mathrm{C}$ (after Schwartz et al., 1977): (O) measured solubility, (__ ) calculated solubility. strength of 0.16 and reported the apparent dissociation constant, $\mathrm{p} K_{\mathrm{a}}$, to be 8.06 and the solubility of the unionized form to be $18.4 \mu \mathrm{g} / \mathrm{ml}$. The solubility of phenytoin can be calculated as a function of $\mathrm{pH}$ from Eqn 4 and is shown in Fig. 1.

Theoretical aspects of crystal growth from solution

The growth of crystals from solution involves a series of steps by which a growth unit passes from the bulk solution to the crystal lattice. They include: transport of growth units from the bulk solution to the crystal surface, adsorption of growth units onto the solid surface, possible diffusion of the growth units from the point of adsorption to a favorable incorporation site and integration into the crystal lattice. The rate of growth is limited by the slowest of these processes.

There are several possible sites for incorporation into the crystal lattice. Interaction between a growth unit and the crystal lattice is greatest at a kink site, where bonds are formed in three directions. Bonds are formed in two directions at step sites and in only one direction at ledge sites. Thus, incorporation into the lattice occurs predominately at kink sites.

The growth unit can be an ion, a single molecule or two or more molecules interacting in solution. During the growth process, solvent growth-unit interactions in solution are replaced by solid-solid bonds in the crystal lattice. Adsorption onto the crystal surface results in a partial desolvation of the growth unit. Incorporation into a kink site then completely replaces the kinksolvent interaction with solid-solid interaction.

The driving force for crystallization, the supersaturation, is the difference in chemical potential of the substance, $i$, in solution, $\mu_{i}$, and in a solution at equilibrium with the solid phase, $\mu_{i, \text { eq }}$ (i.e., a saturated solution):

$\Delta \mu_{i}=\mu_{i}-\mu_{i, \mathrm{eq}}$

Writing the chemical potential as:

$\mu_{i}=\mu_{i}^{\mathrm{o}}+R T \ln a_{i}=\mu_{i}^{\mathrm{o}}+R T \ln \gamma_{i} C_{i}$ 
where $\mu^{\mathrm{o}}{ }_{i}$ is the chemical potential of $i$ in the standard state, and the activity of $i$ in solution, $a_{i}$, is the product of the activity coefficient, $\gamma_{i}$, and the concentration of $i, C_{i}$, Eqn 5 becomes:

$\Delta \mu_{i} / R T=\ln \left(a_{i} / a_{i, \mathrm{eq}}\right)=\ln \left(\gamma_{i} C_{i} / \gamma_{i, \mathrm{eq}} C_{i, \mathrm{eq}}\right)$

If the activity coefficient is not a strong function of concentration, the ratio $\gamma_{i} / \gamma_{i, \mathrm{eq}}$ is close to unity and the supersaturation, $\sigma$, becomes

$\sigma=\Delta \mu / R T \approx \ln \left(C_{i} / C_{i, \mathrm{eq}}\right)$

\section{Crystal growth mechanisms}

The rate-limiting step for crystal growth is frequently assigned to two general categories: diffusion control, and surface integration control. If diffusion of growth units from the bulk solution to the crystal surface is slow compared to integration into the lattice, growth is diffusion controlled. Otherwise, if diffusion is fast compared to integration, the solute concentration at the crystal surface is equal to the bulk concentration and growth is controlled by surface integration. Both of these categories are discussed in further detail below.

The mechanism of crystal growth can be determined by analyzing the predicted growth rate dependence on crystallization conditions and comparison of the predictions with measured kinetic data.

\section{Diffusion control}

In the case of diffusion control, crystal growth is limited by the rate of transport of growth units across a boundary layer to the crystal surface. The crystallization rate would follow Fick's law of diffusion (Ohara and Reid, 1973) and the growth rate, $G$, is proportional to the driving force:

$G=A_{1} \sigma$

In this case the rate constant, $A_{1}$, depends upon the diffusion boundary layer thickness, $\delta$, sur- rounding the crystal and the diffusion rate of the growth units.

\section{Surface integration control}

A large body of theoretical work has been devoted toward the development of models that describe the formation and propagation of kink sites on the crystal surface. If the surface is very rough and covered with kinks, every encounter of a growth unit with the solid is likely to be at a site favorable for incorporation. This type of growth usually occurs at high temperatures and is said to be due to a rough surface mechanism. Growth rate becomes a linear function of the driving force, $\sigma$ (Weeks and Gilmer, 1979), as:

$G=A_{2} \sigma$

Crystal growth occurring by this mechanism tends to result in rounded, non-facetted crystals.

At lower temperatures, crystal growth occurs by a layer mechanism; growth units are integrated at kink sites on a flat crystal surface. Two mechanisms have been proposed as the source of kink sites, as described below.

Two-dimensional nucleation where nuclei form on the crystal and spread across the flat surface

This process is somewhat analogous to homogenous nucleation. Clusters of growth units continually form and dissolve on the crystal surface. When the radius of a cluster exceeds a critical value, $r^{*}$, the excess free energy of the cluster is at a maximum, $\Delta G^{*}$, and the nucleus can grow. As with homogeneous nucleation, the rate of clusters attaining the critical size is strongly dependent upon supersaturation, being essentially zero at low supersaturation and rapidly increasing to a constant value at higher supersaturation.

There are two main models describing two-dimensional nucleation: mononuclear models, where only one nuclei can form on a given crystal face, and birth and spread or polynuclear models, where more than one nuclei can form on a face and where nuclei can form on top of other growing nuclei. 
Numerous equations have been derived to describc the dependence of growth rate on supersaturation for the two-dimensional nucleation mechanism. The one used here was developed by Gilmer and Bennema (1972) for the birth and spread model and is:

$G=B_{1}(\Delta \mu / R T)^{5 / 6} \cdot\left(e^{-(1 / 3) \Delta G^{*} / k T}\right)$

where: $\Delta \mu / R T$ is the driving force, $T$ denotes temperature, $R$ is the gas constant and $k$ the Boltzmann constant.

In Eqn $11 \mathrm{a}$, the term $\Delta G^{*} / k T$ can be expressed in terms of the driving force, $\Delta \mu / R T$, as:

$\Delta G^{*} / k T=D\left(\gamma^{\prime} / k T\right)^{2} /(\Delta \mu / R T)$

where $\gamma^{\prime}$ is the edge free energy per growth unit at the edge of a critical nucleus and $D$ is equal to $\pi$ for circular clusters and 4 for square clusters.

Spiral growth, where kink sites are continually formed by the attachment of growth units to a screw dislocation

This mechanism was developed by Burton, Cabrera and Frank (1951) to explain experimentally observed crystal growth rates at low supersaturation; where the two-dimensional nucleation growth mechanism had predicted no growth. A self propagating spiral provides the necessary kink sites for growth unit incorporation and the BCF theory elegantly describes the observed growth kinetics.

Growth spirals are a result of dislocations in the crystal structure. The dislocations produce steps at the crystal surface and attachment of growth units to the steps produces a self-sustaining spiral.

Growth spirals have been observed in a variety of systems. In fact, because most crystals are imperfect and have dislocations, the spiral growth mechanism is expected to be the dominant mechanism at low supersaturation (Bennema and Van der Eerden, 1987).

The classical expression for spiral growth is:

$$
\begin{gathered}
G=C_{1} \Delta \mu / R T \cdot\left(e^{\Delta \mu / R T}-1\right) \\
\tanh \left(C_{2} /(\Delta \mu / R T)\right)
\end{gathered}
$$

Eqn 13 can be simplified in the moderately low supersaturation region where the term $C_{2} /$ $(\Delta \mu / R T)$ is small (Ohara and Reid, 1972) as:

$G=C_{1} \cdot \Delta \mu / R T \cdot\left(e^{\Delta \mu / R T}-1\right)$

The value of the constant $C_{1}$ in Eqn $14 \mathrm{a}$ is proportional to $e^{-\Delta G_{\text {desolv }} / R T}$, where $\Delta G_{\text {desolv }}$ is the molar activation-free energy of desolvation of a growth unit (Bennema and Gilmer, 1973).

If diffusion resistance from the bulk to the crystal surface is negligible, then the three remaining crystal growth mechanisms each dominate in a particular supersaturation region. At low supersaturation growth will be by a screw dislocation mechanism. At intermediate supersaturation spiral growth and birth and spread mechanisms may both be present. However, at somewhat higher supersaturation the two-dimensional nucleation mechanism will prevail. At very high supersaturation, the radius of a critical nucleus becomes close to the diameter of a growth unit and a rough surface growth mechanism will result.

\section{Materials and Methods}

\section{Materials}

Phenytoin free acid, 5,5-diphenylhydantoin (Sigma lot no. 12F-0177) was used as provided by the manufacturer for all experimental work. All other chemicals used for the preparation of solutions were of reagent grade. The water used in this study was filtered through a double-deionized purification system (Milli $Q$ Water System from Millipore Co.). Phosphate buffer at $\mathrm{pH}$ 2.2 was prepared by either mixing equal volumes of $0.10 \mathrm{M} \mathrm{NaH} \mathrm{PO}_{4}$ and $0.10 \mathrm{M} \mathrm{H}_{3} \mathrm{PO}_{4}$ and adjusting the ionic strength to 0.15 with $\mathrm{NaCl}$, or by addition of $0.10 \mathrm{M} \mathrm{NaOH}$ to $0.10 \mathrm{M} \mathrm{H}_{3} \mathrm{PO}_{4}$ until reaching $\mathrm{pH} 2.2$ and adjusting the ionic strength to 0.15 with $\mathrm{NaCl}$. No change in results was observed between these buffer solutions. The

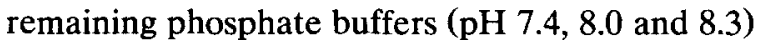
were made at ionic strength 0.15 following Sørenson's buffer system. All buffer solutions were filtered with a $0.45 \mu \mathrm{m}$ membrane filter prior to use. 


\section{Assay and identification}

Phenytoin free acid was identified as the sole crystallization product by confirmation of the melting point at $296^{\circ} \mathrm{C}$ with a Dupont 10990 thermal analyzer. Phenytoin concentration in solution was measured by UV spectrophotometry with a Beckman DU-8 spectrophotometer at 258 nm.

\section{Crystallization procedure}

Crystallization experiments were conducted at constant temperature in a contoured-bottom jacketed glass vessel. Phenytoin crystal growth rate was measured as a function of supersaturation at $25^{\circ} \mathrm{C}$ in $\mathrm{pH} 2.2,7.4,8.0$ and 8.3 phosphate buffer and at $45^{\circ} \mathrm{C}$ in $\mathrm{pH} 2.2,7.4$ and 8.0 phosphate buffer. Agitation was provided by a propeller-type glass impeller at 400 and $1600 \mathrm{rpm}$. Temperature control was maintained at 25 or $45^{\circ} \mathrm{C}$ with the use of an external water bath.

The crystallization vessel was located directly within the sample stand of a Coulter Counter Multisizer particle analyzer and crystal size distribution (CSD) measured in situ with a $140 \mu \mathrm{m}$ diameter orifice.

In an experiment, a known amount of buffer (100-150 ml) was placed in the crystallizer and the agitation rate and temperature of the buffer adjusted to the desired levels. Production of phenytoin seed crystals and creation of a supersaturated solution for their growth was accomplished by addition of a small amount of a highly concentrated phenytoin solution to the crystallizer. The phenytoin solution contained 6-12 $\mathrm{mg} / \mathrm{g}$ phenytoin (free acid) dissolved in $0.10 \mathrm{M}$ $\mathrm{NaOH}$. The small amount of solution added (typically less than $1 \mathrm{~g}$ to $100 \mathrm{ml}$ of buffer) was not sufficient to change either the $\mathrm{pH}$ or the ionic strength of the buffer. Phenytoin crystals nucleated immediately upon contact between the phenytoin solution and the buffer. However, the extent of nucleation was not sufficient to deplete the excess phenytoin from solution and the seed crystals then grew from the residual supersaturation. The crystal size distribution and solution concentration were then monitored throughout the experiment.

\section{Growth rate analysis}

Phenytoin crystal growth rate, $G$, was evaluated from the increase in size, $\Delta L$, of the cumulative number distribution, $F$, with time, $\Delta t$ (Misra and White, 1973) as:

$G=(\mathrm{d} L / \mathrm{d} t)_{F} \approx(\Delta I / \Delta t)_{F}$

If crystal growth rate is not size dependent, or if there is no dispersion of growth rates, successive cumulative number profiles will be parallel and crystal growth rate can be equivalently cvaluated at any value of cumulative number, $F$. The growth rate was evaluated at several $F$ values to provide an average change between measured distributions and to verify that crystal growth rate was not size dependent.

\section{Results and Discussion}

Batch crystallization results and growth mechanism evaluation

The nucleation of phenytoin crystals upon contact of the high concentration phenytoin solution

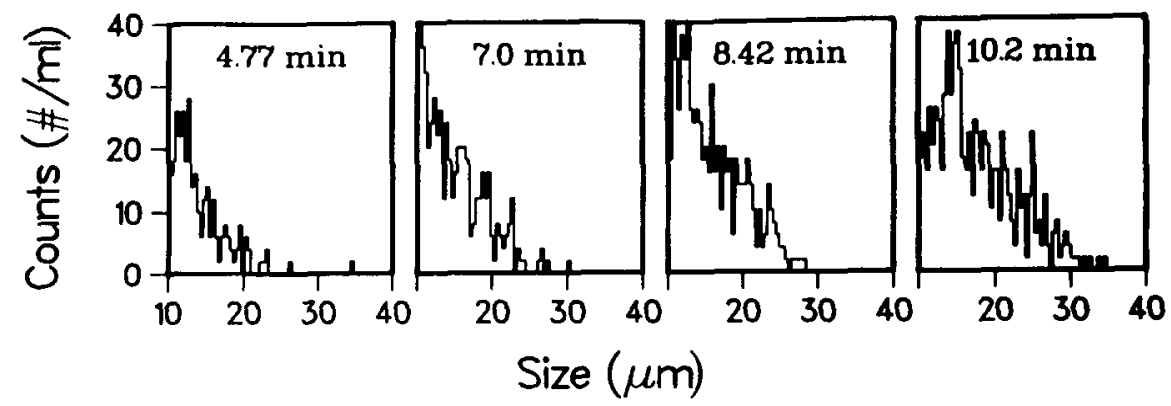

Fig. 2. Differential plots of phenytoin crystal size distribution measured in a batch crystallization expcriment. 


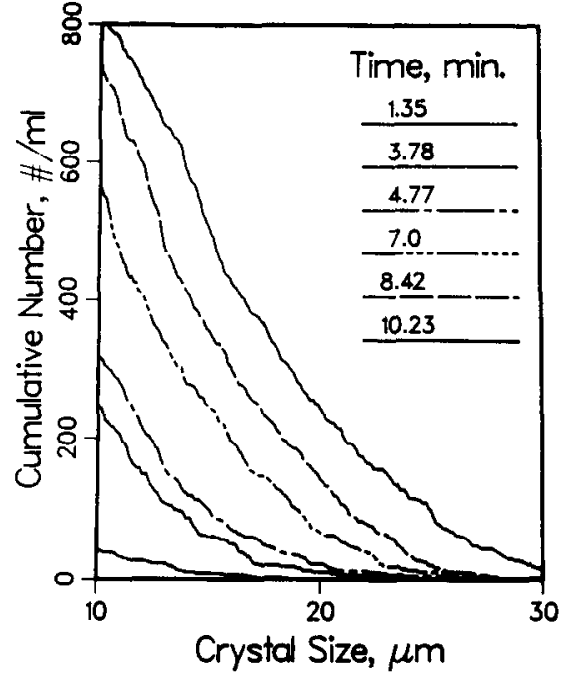

Fig. 3. Cumulative number representation of the CSDs presented in Fig. 2.

with the buffer in the crystallizer produced a narrow size distribution of crystals with no additional nucleation after mixing. A sample of CSDs measured during an experiment is shown in Fig. 2. Each subplot of Fig. 2 is a differential plot of the number of counts accumulated in each channel of the Coulter Counter Multisizer from a $1 \mathrm{ml}$ sample. The average value of the size interval for each channel has been used for the $x$-axis. The same crystal distributions are displayed as cumulative number profiles in Fig. 3. The change in phenytoin concentration measured during the experiment is shown in Fig. 4.

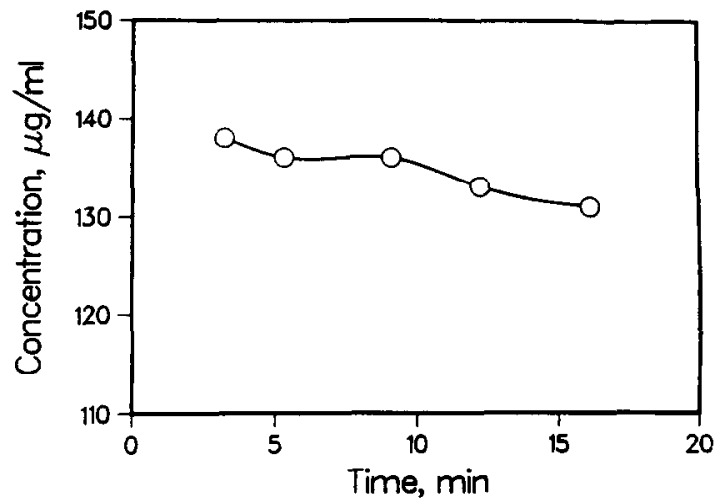

Fig. 4. Phenytoin concentration profile measured during a batch crystallization experiment.

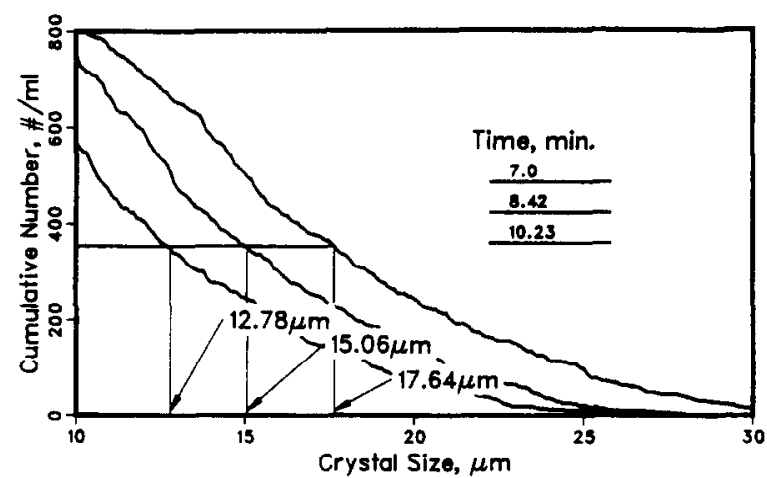

Fig. 5. Analysis of cumulative number profiles used to evaluate phenytoin growth rate.

An example of growth rate analysis is shown in Fig. 5. Phenytoin growth rate was evaluated at a cumulative number value of $F=380 \mathrm{ml}^{-1}$ from the CSD measurements at 7.0, 8.42 and 10.23 min. The sizes corresponding to the cumulative number profile at $380 \mathrm{ml}^{-1}$ for these three samples are $12.78,15.06$ and $17.64 \mu \mathrm{m}$. Thus, the average growth rate between 7.0 and $8.42 \mathrm{~min}$ is $1.61 \mu \mathrm{m} / \mathrm{min}$ and between 8.42 and $10.23 \mathrm{~min}$, $1.42 \mu \mathrm{m} / \mathrm{min}$. Note that during the time intervals there was only a slight change in phenytoin concentration.

Phenytoin growth rate was evaluated using the above procedure at eight cumulative number values from 20 to $600 \mathrm{ml}^{-1}$ and is shown as a function of cumulative number in Fig. 6. There is some scatter in the calculated growth rates at low values of $F$ but this is to be expected as the number of crystals contributing to the analysis is

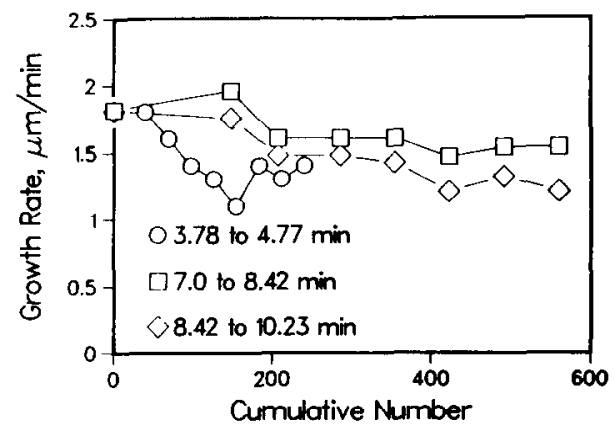

Fig. 6. Phenytoin crystal growth rate vs cumulative number value. 
small. At intermediate and high values of $F$ the growth rate is independent of size and a representative numerical average is obtained.

\section{Phenytoin crystal growth mechanism}

The mechanism controlling addition of phenytoin growth units to the crystal lattice may be elucidated by comparison of the measured growth kinetics with the four models described previously.

In Fig. 7a, phenytoin growth rate measured in pH 2.2 phosphate buffer at 25 and $45^{\circ} \mathrm{C}$ is plotted vs supersaturation. There is a strong dependence of growth rate on supersaturation and clearly the two models that predict a linear dependence of growth rate on supersaturation (rough surface and diffusion control) do not apply. A rough surface growth mechanism is also contradicted by the appearance of the phenytoin crystals, which are faceted without rounding of any edges. Diffusion resistance to growth is negligible since no change in measured growth kinetics was observed when the agitation rate was increased from 400 to $1600 \mathrm{rpm}$. Similar observations were also made of phenytoin growth kinetics measured at both temperatures in $\mathrm{pH} 7.4,8.0$ and 8.3 phosphate buffer (Fig. $7 b$ and $c$ ).

An indication of which of the two remaining mechanisms best describes phenytoin growth can be obtained by comparison of the expected growth dependence on supersaturation with that found from experimental measurements. In the case of a screw dislocation mechanism, Eqn 14a can be written in terms of the supersaturation as:

$G=C_{1} \cdot \sigma \cdot\left(e^{\sigma}-1\right)$

and a plot of measured phenytoin growth rates, $G$, vs $\sigma \cdot\left(e^{\sigma}-1\right)$ would be linear if the screw dislocation mechanism is present.

Indeed, the spiral growth model describes phenytoin growth kinetics well as shown by the linear relationships found in Fig. $8 \mathrm{a}(\mathrm{pH} \mathrm{2.2),} \mathrm{b}$ (pH 7.4) and $\mathrm{c}(\mathrm{pH} 8.0$ and 8.3). Note that there is some deviation from linearity at high values of $\sigma \cdot\left(e^{\sigma}-1\right)$ in Fig. 8c. This may indicate the beginning of a transition from a screw dislocation growth mechanism to a combination of screw dislocation and two-dimensional nucleation growth mechanisms.

A linear regression of the data presented in Fig. $8 \mathrm{a}-\mathrm{c}$ was used to obtain the values of slope and intercept presented in Table 1. According to Eqn $14 \mathrm{~b}$, the intercept of a plot of $G$ vs $\sigma \cdot\left(e^{\sigma}-\right.$ 1) should be zero. This was the case for all results at $45^{\circ} \mathrm{C}$. However, from the $25^{\circ} \mathrm{C}$ results the intercept is found to be significantly $(p \leqq 0.05)$ different from zero. This is most probably an artifact of growth rate measurement and does not indicate a lack of fit of the model as the predicted dependence of growth rate on supersaturation agrees well with the observed results. The two-parameter model for spiral growth (Eqn 13) does not provide a significantly better description of our data. The estimated value of $C_{2}$ is approx. 10 . Thus, the fourth term in Eqn $13, \tanh \left(C_{2} /(\Delta \mu /\right.$ $R T)$ ), is approximately unity and can be neglected.

An estimate of the desolvation energy for phenytoin crystal growth can be made from the temperature dependence of the constant $C_{1}$. Since $C_{1}$ is proportional to $\Delta G_{\text {desolv }} / R T$, an Arrhenius plot of $\ln C_{1}$ vs $1 / T$ would have a slope of $-\Delta G_{\text {desolv }} / R$. The estimates of $\Delta G_{\text {desolv }} / R$ (Table 1) are much higher than would be expected if diffusion was the rate-limiting step of phenytoin crystal growth and confirm a surface-controlled process.

The birth and spread model (Eqn 11a) can be expressed as:

$G=B_{1} \cdot \sigma^{5 / 6} \cdot\left(e^{-B_{2} / \sigma}\right)$

where $B_{2}=D / 3 \cdot\left(\gamma^{\prime} / k T\right)^{2}$. If phenytoin growth is via a two-dimensional nucleation mechanism a plot of $(\ln G-5 / 6 \ln \sigma)$ vs $1 / \sigma$ should yield a linear relationship with slope equal to $-B_{2}$ and intercept equal to $\ln B_{1}$. There is evidence of a linear relationship at high supersaturation values (low $1 / \sigma$ values) in Fig. 9a-c. However, there is deviation from linearity at low supersaturation values (high $1 / \sigma$ ) at all $\mathrm{pH}$ values and temperatures except at $\mathrm{pH} 8.0,45^{\circ} \mathrm{C}$ where the scatter in the data is large.

The values of $B_{1}$ and $B_{2}$ resulting from a 

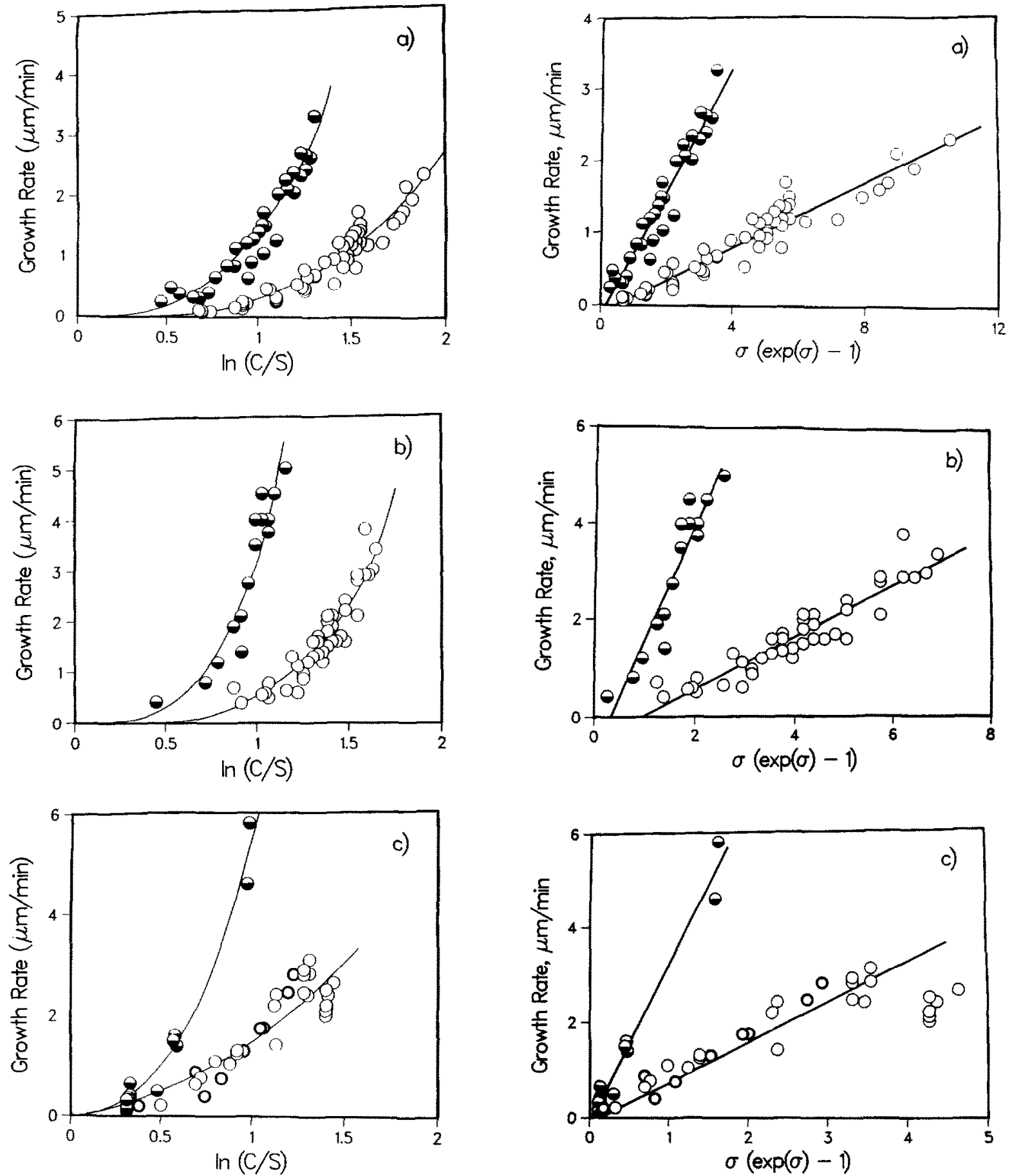

Fig. 7. Phenytoin crystal growth rate as a function of supersaturation and temperature: (o) $25^{\circ} \mathrm{C}$, (O) $45^{\circ} \mathrm{C}$; (a) pH 2.2 ; (b)

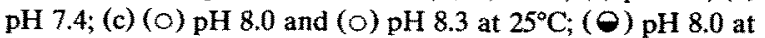
$45^{\circ} \mathrm{C}$. The curves were constructed according to Eqn 16 from the parameters presented in Table 3 .

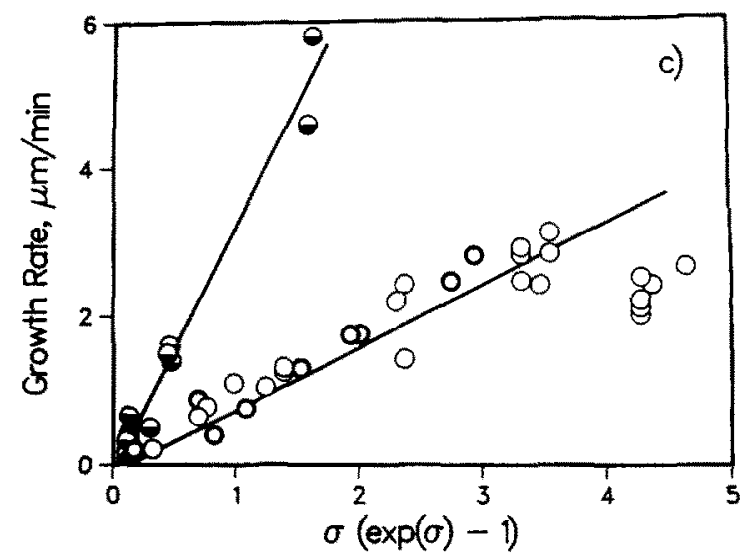

Fig. 8. Test of the screw-dislocation model (Eqn 14b) for phenytoin crystal growth: (o) $25^{\circ} \mathrm{C}$, (Ө) $45^{\circ} \mathrm{C}$; (a) pH 2.2 ; (b)

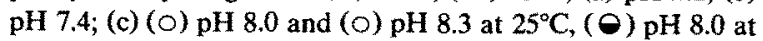
$45^{\circ} \mathrm{C}$. 
TABLE 1

Calculated values of slope and intercept for the spiral growth mechanism (Eqn 14b)

\begin{tabular}{lllll}
\hline $\mathrm{pH}$ & $\begin{array}{l}\text { Temperature } \\
\left({ }^{\circ} \mathrm{C}\right)\end{array}$ & Intercept & $C_{1}$ & $\begin{array}{l}\Delta G_{\text {desolv }} \\
(\mathrm{kJ} / \mathrm{mol})\end{array}$ \\
\hline 2.2 & 25 & -0.10 & 0.23 & 52 \\
& 45 & $-0.14^{\mathrm{a}}$ & 0.85 & \\
7.4 & 25 & $-0.52^{\mathrm{a}}$ & 0.54 & 58 \\
& 45 & $-0.78^{\mathrm{a}}$ & 2.38 & \\
8.0 & 25 & $0.097^{\mathrm{b}}$ & $0.79^{\mathrm{b}}$ & 57 \\
& 45 & $-0.10^{\mathrm{a}}$ & 3.30 &
\end{tabular}

${ }^{a}$ Intercept not significantly different from $0.0(p \leq 0.05)$.

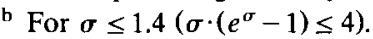

linear regression of the kinetic data, the value of $\gamma^{\prime} / k T$ (found from $B_{2}$ with $D / 3=3.6$ ) and an estimate of the desolvation energy, $\Delta G_{\text {desolv }}$ (found from the temperature dependence of $B_{1}$ ) are presented in Table 2 .

We can test the two-dimensional nucleation model further by comparing an estimate of $\gamma^{\prime} / k T$ of 1.0-5.1kT (from Bennema and Söhnel, 1990) with the $\gamma^{\prime} / k T$ values calculated from the twodimensional nucleation model. The $\gamma^{\prime} / k T$ values in Table 2 fall slightly below and within the low end of the range of the estimates. The calculated edge free energy would increase slightly if the maximum value of $1 / \sigma$ used in the linear regression was reduced. However, given the uncertainty in the method used to estimate the value of $\gamma^{\prime} / k T$, this is not necessary as the calculated and estimated values are reasonably close.

Frequently, crystal growth data is represented by a power law relationship as:

$G=k_{\mathrm{g}} \sigma^{a}$

where $k_{\mathrm{g}}$ is the growth rate constant and $a$ the supersaturation exponent. The values of $k_{\mathrm{g}}$ and $a$ were computed by a non-linear regression of $G$ vs $\sigma$ and are presented with their standard deviations in Table 3. These parameters were then used to construct the curves in Fig. 7a-c. While Eqn 16 is strictly an empirical relationship, the value of the supersaturation exponent, $a \approx 2$, has been assumed to indicate a screw dislocation mechanism and a value $a \geqq 2$ to indicate a two-
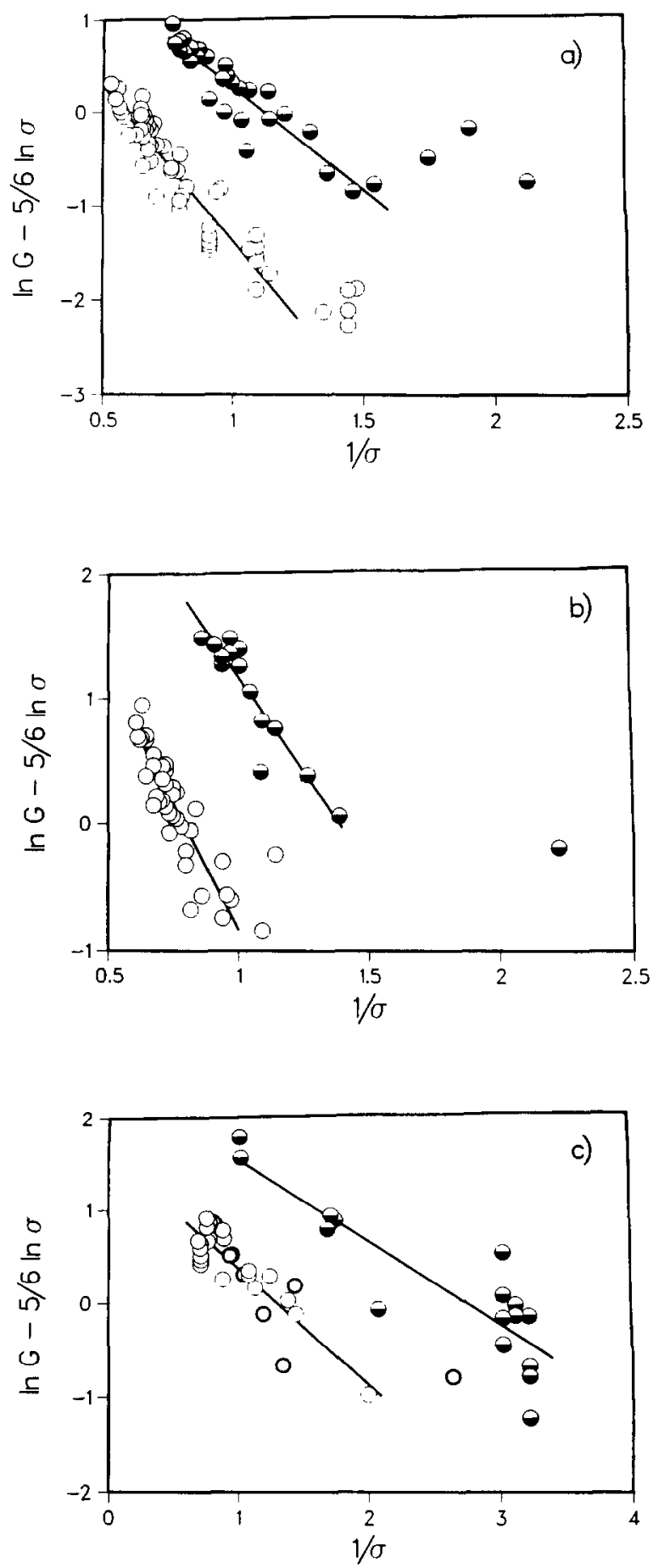

Fig. 9. Test of the two-dimensional nucleation model (Eqn 11b) for phenytoin crystal growth: (O) $25^{\circ} \mathrm{C}$, ( $\left.\odot\right) 45^{\circ} \mathrm{C}$; (a) $\mathrm{pH}$ 2.2; (b) $\mathrm{pH} 7.4$; (c) (O) $\mathrm{pH} 8.0$ and (O) $\mathrm{pH} 8.3$ at $25^{\circ} \mathrm{C}$, ( $\left.\odot\right)$ pH 8.0 at $45^{\circ} \mathrm{C}$. 
TABLE 2

Calculated values of intercept and slope for the two-dimensional growth mechanism (Eqn 11 b)

\begin{tabular}{llllll}
\hline pH & $\begin{array}{l}\text { Temperature } \\
\left({ }^{\circ} \mathrm{C}\right)\end{array}$ & $B_{2}$ & $\ln B_{1}$ & $\gamma^{\prime} / k T$ & $\begin{array}{l}\Delta G_{\text {desolv }} \\
(\mathrm{kJ} / \mathrm{mol})\end{array}$ \\
\hline 2.2 & 25 & 2.67 & 1.47 & 1.5 & \\
& 45 & 1.26 & 1.57 & 1.0 & \\
& $25^{\mathrm{a}}$ & 3.30 & 1.93 & 1.7 & 20 \\
& $45^{\mathrm{b}}$ & 2.19 & 2.45 & 1.4 & \\
7.4 & 25 & 3.07 & 2.46 & 1.6 & \\
& 45 & 1.40 & 2.54 & 1.1 & \\
& $25^{\mathrm{c}}$ & 4.0 & 3.16 & 1.8 & 41 \\
& $45^{\mathrm{d}}$ & 3.0 & 4.2 & 1.6 & \\
& 25 & 0.99 & 1.38 & 0.91 & \\
& $25^{\mathrm{e}}$ & 1.25 & 1.62 & 0.86 & 32 \\
& $45^{2}$ & 0.89 & 2.43 & 0.86 &
\end{tabular}

a $1 / \sigma \leq 1.25 ;{ }^{\mathrm{b}} 1 / \sigma \leq 1.5 ;{ }^{\mathrm{c}} 1 / \sigma \leq 1.0 ;{ }^{\mathrm{d}} 1 / \sigma \leq 1.5 ;{ }^{\mathrm{e}} 1 / \sigma$ $\leq 2.0$.

dimensional nucleation mechanism. The only results with a supersaturation exponent close to the expected value of two (for the spiral growth model) are those from $\mathrm{pH} 8.0$ and 8.3 solutions. Yet, under these conditions, a reasonable fit with the two-dimensional nucleation model was found. This illustrates some of the difficulties encountered when attempting to elucidate crystal growth mechanisms from kinetic data. Numerous expressions can be found for surface controlled growth mechanisms and all may describe the kinetic data with varying degrees of accuracy.

Solubility considerations in phenytoin growth kinetics and mechanism

At constant temperature, and in the $\mathrm{pH}$ range of 2.2-8.0, phenytoin growth rate increases with

TABLE 3

Calculated values of $k_{g}, a$ and their standard deviations for the power law model (Eqn 16)

\begin{tabular}{llllll}
\hline $\mathrm{pH}$ & $\begin{array}{l}\text { Temperature } \\
\left({ }^{\circ} \mathrm{C}\right)\end{array}$ & $\begin{array}{l}k_{\mathrm{g}} \\
(\mu \mathrm{m} / \mathrm{min})\end{array}$ & SD & $a$ & $\mathrm{SD}$ \\
\hline 2.2 & 25 & 0.29 & 0.02 & 3.2 & 0.2 \\
& 45 & 1.3 & 0.05 & 3.1 & 0.2 \\
7.4 & 25 & 0.45 & 0.05 & 4.0 & 0.3 \\
& 45 & 3.2 & 0.14 & 3.5 & 0.4 \\
8.0 & 25 & 1.5 & 0.09 & 1.7 & 0.2 \\
& 45 & 5.6 & 0.20 & 2.5 & 0.2 \\
\hline
\end{tabular}

TABLE 4

Calculated values of the $\alpha$-factor for $25^{\circ} \mathrm{C}$ phenytoin solutions

\begin{tabular}{llcclr}
\hline $\mathrm{pH}$ & $\begin{array}{l}C_{\mathrm{s}} \\
(\mu \mathrm{g} / \mathrm{ml})\end{array}$ & $\begin{array}{l}\% \\
\text { ionized }\end{array}$ & $\ln x_{\mathrm{s}}$ & $\begin{array}{l}\Delta H_{\mathrm{s}} \\
(\mathrm{kJ} / \mathrm{mol})\end{array}$ & $\alpha^{\mathrm{c}}$ \\
\hline 2.2 & 18.4 & 0 & -9.5 & $19.6^{\mathrm{a}}$ & 10 \\
5.4 & 18.4 & 0 & -9.5 & $31.3^{\mathrm{b}}$ & 6 \\
7.4 & 22.4 & 18 & -9.3 & $33.6^{\mathrm{b}}$ & 7 \\
7.4 & 22.4 & 18 & -9.3 & $19.4^{\mathrm{a}}$ & 10 \\
8.0 & 34.4 & 47 & -8.0 & $19.0^{\mathrm{a}}$ & 10 \\
8.3 & 50.4 & 64 & -8.5 & $18.6^{\mathrm{a}}$ & 9 \\
\hline
\end{tabular}

a From Eqn 18, $\Delta H_{\text {fusion }}=47.5 \mathrm{~kJ} / \mathrm{mol}, T_{\text {fusion }}=294^{\circ} \mathrm{C}$.

b From Schwartz et al. (1977).

c $n_{\mathrm{s}} / n_{1}=0.5$.

increasing $\mathrm{pH}$. For example, at $25^{\circ} \mathrm{C}$ and supersaturation of 1 , the growth rate constant, $k_{\mathrm{g}}$, is 0.29 at $\mathrm{pH} 2.2,0.45$ at $\mathrm{pH} 7.4$ and 1.5 at $\mathrm{pH}$ 8.0. However, no further increase in growth rate was observed when the $\mathrm{pH}$ was increased from 8.0 to 8.3. (The $\mathrm{pH} 8.3$ results are not significantly different from the $\mathrm{pH} 8.0$ results and both were grouped together as $\mathrm{pH} 8.0$ for analysis of model constants.)

The increase in growth rate with $\mathrm{pH}$ is probably due to the increase in phenytoin solubility with $\mathrm{pH}$ (solubility values are presented in Table 4). In the spiral growth model the constant, $C_{1}$ (Eqn 14b), includes $N_{0}$, a solubility parameter. Thus, at constant supersaturation and temperature, an increase in solubility (i.e., pH) would result in a corresponding increase in growth rate (Boistelle and Astier, 1988).

The mechanism of phenytoin crystal growth can be predicted from solubility considerations. Solubility affects crystal growth by changing the roughness of the solid-fluid interface. The concept of surface roughening was originally formulated by Jackson (1958) to describe solid-liquid interactions during crystal growth from the melt. Jackson's $\alpha$-factor is a measure of the roughness of the growing crystal surface and thus, an indication of the crystal growth mechanism. Jackson's work was extended to crystal growth in solution by Bennema and Gilmer (1973). They showed that the $\alpha$-factor can be written as:

$\alpha=\left(n_{\mathrm{s}} n_{\mathrm{t}}\right) \cdot\left(\Delta H_{\text {solution }} / R T\right)$ 
where $n_{\mathrm{s}}$ is the number of bonds formed with a growth unit in the growth plane, $n_{\mathrm{t}}$ denotes the total number of bonds in the bulk crystal and $\Delta H_{\text {solution }}$ is the heat of solution. If the heat of solution is not known, it can be estimated from the heat of fusion, $\Delta H_{\text {fusion }}$, the melting temperature, $T_{\text {fusion }}$, and the solubility, $x_{\mathrm{s}}$ (mole fraction), as:

$$
\Delta H_{\text {solution }} / R T=\left(\Delta H_{\text {fusion }} / R T_{\text {fusion }}\right)-\ln x_{\mathrm{s}} .
$$

The three growth mechanisms of interest here: rough surface, two-dimensional nucleation and spiral growth are predicted by the magnitude of the $\alpha$-factor. A low value $(\alpha \leqq 3.2)$ corresponds to a rough surface where growth is continuous, an intermediate value $(3.2 \leqq \alpha \leqq 4.0)$ indicates a smoother interface with crystal growth dominated by two-dimensional nucleation mechanisms, and a high value $(\alpha \geqq 4.0)$ indicates a very smooth interface where growth is due to screw dislocations in the crystal.

The $\alpha$-factor was calculated for phenytoin crystal growth from the thermodynamic and solubility data provided by Schwartz et al. (1977) and the results are presented in Table 4. The high $\alpha$-factor values indicate that phenytoin crystals may grow by a spiral growth mechanism.

\section{Other effects on phenytoin crystal growth}

\section{Solvent effects}

Phenytoin crystallization kinetics may also be influenced by solvent effects. The degree of ionization of the phenytoin molecules and the concentration of the phosphate species $\mathrm{H}_{3} \mathrm{PO}_{4}$, $\mathrm{H}_{2} \mathrm{PO}_{4}^{-}$and $\mathrm{HPO}_{4}^{2-}$, changes with $\mathrm{pH}$ as illustrated in Fig. 10. At pH 2.2, the phosphate species $\mathrm{H}_{3} \mathrm{PO}_{4}$ and $\mathrm{H}_{2} \mathrm{PO}_{4}^{-}$are present in equal proportions and phenytoin is completely unionized. At $\mathrm{pH} 8.0$, the phosphate species present are $\mathrm{H}_{2} \mathrm{PO}_{4}^{-}$ and $\mathrm{HPO}_{4}^{2-}(14 \% / 86 \%)$ and phenytoin is nearly $50 \%$ ionized. Interaction between the buffer and the growing crystal is not necessarily the same for all species (Boistelle and Astier, 1988) and this may affect growth kinetics.

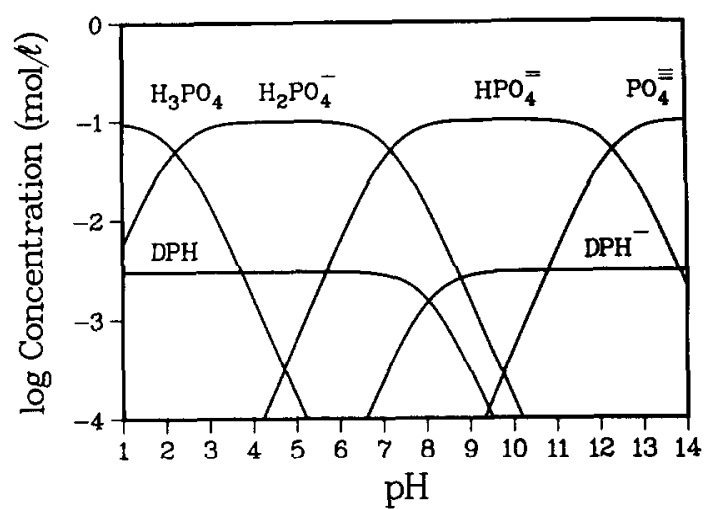

Fig. 10. Concentration of phenytoin and phosphate buffer species as a function of $\mathrm{pH}(0.1 \mathrm{M}$ buffer concentration and $0.003 \mathrm{M}$ phenytoin concentration).

\section{Self-association}

Phenytoin was shown to form cyclic dimers in solution by Sohár (1968). However, the orientation of the phenytoin molecules in the dimer is not the same as that found in the crystal. Therefore, the dimers cannot be incorporated into the lattice and their presence effectively reduces the supersaturation. If dimcrization betwecn ionized phenytoin molecules does not occur as readily as between unionized molecules a slower growth rate at low $\mathrm{pH}$ would be expected.

\section{Buffer capacity}

During crystal growth of a weak acid such as phenytoin, incorporation of growth units into the lattice could result in a depletion of hydrogen ions at the solid/solvent interface. The hydrogen ions would be removed by the reaction $\mathrm{H}^{+}$ $+(\mathrm{DPH})^{-} \rightleftharpoons \mathrm{DPH}$ which occurs when ionized phenytoin molecules are incorporated into the solid. In a buffered solution the change in hydrogen ion concentration is compensated for by a shift in the reaction: $\mathrm{H}_{3} \mathrm{PO}_{4} \rightleftharpoons \mathrm{H}^{+}+\mathrm{H}_{2} \mathrm{PO}_{4}^{-}$. However, the buffering capacity of the $\mathrm{pH} 8.0$ and 8.3 solutions is lower than that of the $\mathrm{pH} 2.2$ and 7.4 solutions. If the growth rate was sufficiently rapid to cause a localized depletion of $\mathrm{H}^{+}$, a concentration gradient of $\mathrm{H}^{+}$would exist between the crystal surface and the bulk solution. Thus, it is possible that the $\mathrm{pH}$ at the crystal/ solution interface could be greater than that in 
the bulk solution. A higher $\mathrm{pH}$ would increase the phenytoin solubility at the interface and decrease the supersaturation at that point. This would lead to a weaker dependence of growth rate on supersaturation and may explain the similarity between the $\mathrm{pH} 8.0$ and 8.3 results.

The existence of a localized $\mathrm{pH}$ profile at the solid/liquid interface has been used to explain the observed change in dissolution rates of weak acids and weak bases in buffered and unbuffered solutions (Mooney et al., 1981a,b; McNamara and Amidon, 1986). This effect would be negligible at $\mathrm{pH} 2.2$ where phenytoin is completely unionized. However, as the $\mathrm{pH}$ approaches the $\mathrm{p} K_{\mathrm{a}}$ of 8.06 , nearly half of the phenytoin molecules are ion-

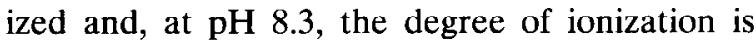
$63 \%$. Thus, while the overall kinetics were not influenced by agitation rate, the poor buffering capacity of the pH 8.3 solution and the large percentage of phenytoin molecules ionized at that $\mathrm{pH}$ may jointly contribute to the low growth rate.

\section{Conclusion}

Phenytoin crystal growth is controlled by a surface integration mechanism. No dependence of growth kinetics on agitation rate was found and estimates of the desolvation energy are much higher than would be expected for a diffusively controlled process.

The screw dislocation model best describes the dependence of phenytoin crystal growth rate on supersaturation. A two-dimensional nucleation mechanism may also be present at high $\mathrm{pH}$ and temperature although this is unlikely given the high $\alpha$ value (i.e., low phenytoin solubility) calculated for these conditions.

The increase in phenytoin growth rate with $\mathrm{pH}$ (from $\mathrm{pH} 2.2$ to 8.0 ) is due to the increase in solubility with $\mathrm{pH}$. However, a solubility effect contradicts the observed similarity between growth rates measured at $\mathrm{pH} 8.0$ and 8.3 . This may be a consequence of the interaction of different buffer species with the growing crystal surface or the self-association of phenytoin molecules in solution.

\section{Acknowledgments}

This work was partially supported by the National Science Foundation (Grant RII-8613745), the Pharmaceutical Manufactures Association Foundation (Advanced Predoctoral Fellowship in Pharmaceutics) and the Julia E. Emanuel Fellowship from the College of Pharmacy, University of Michigan.

\section{References}

Bennema, P. and Van der Eerden, J.P., Crystal graphs, connected nets, roughening transitions and the morphology of crystals. In Sunagawa, I. (Ed.), Morphology of Crystals, Terra, Tokyo, 1987, p. 18.

Bennema, P. and Gilmer, G.H., Kinetics of crystal growth. In Hartman, P (Ed.), Crystal Growth: An Introduction, North-Holland, Amsterdam, 1973, p. 263.

Bennema, P. and Söhnel, O., Interfacial surface tension for crystallization and precipitation from aqueous solutions. $J$. Crystal Growth, 102 (1990) 547-556.

Boistelle, R. and Astier, J.P., Crystallization mechanisms in solution. J. Crystal Growth, 90 (1988) 14-30.

Burton, W.K., Cabrera, N. and Frank, F.C., The growth of crystals and the equilibrium structures of their surfaces. Phil. Trans. Roy. Soc., A243 (1951) 299-358.

Gilmer, G.H. and Bennema, P., Simulation of crystal growth with surface diffusion. J. Appl. Phys., 43 (1972) 1347-1360.

Higuchi, W.I., Mir, N.A., Parker, A.P. and Hamlin, W.E., Dissolution kinetics of a weak acid, 1,1-hexamethylene p-tolylsulfonylsemicarbazide, and its sodium salt. J. Pharm. Sci., 54 (1965) 8-11.

Jackson, K.A., Liquid Metals and Solidification, Am. Soc. Metals, Cleveland, 1958, p. 174.

McNamara, D.P. and Amidon, G.L., Dissolution of acidic and basic compounds from the rotating disk: Influence of convective diffusion and reaction. J. Pharm. Sci., 75 (1986) 858-868.

Misra, C. and White, E.T., Kinetics of crystallization of aluminum trihydroxide from seeded caustic aluminate solutions. AIChE J., 67 (1971) 53-65.

Mooney, K.G., Mintun, M.A., Himmelstein, K.J. and Stella, V.J., Dissolution kinetics of carboxylic acids: I. Effect of pH under unbuffered conditions. J. Pharm. Sci., 70 (1981) 13-22.

Mooney, K.G., Mintun, M.A., Himmelstein, K.J. and Stella, V.J., Dissolution kinetics of carboxylic acids: II. Effect of buffers. J. Pharm. Sci., 70 (1981) 22-32.

Newton, D.W. and Kluza, R.B., Prediction of phenytoin solubility in intravenous admixtures: Physicochemical theory. Am. J. Hosp. Pharm., 37 (1980) 1647-1651.

Ohara, M. and Reid, R.C., Modeling Crystal Growth Rates from Solution, Prentice-Hall, Englewood Cliffs, NJ, 1973, pp. 47-48. 
Salem, B.R., Yost, R.L., Torosian, G., Davis, F.T. and Wilder, B.J., Investigation of the crystallization of phenytoin in normal saline. Drug Intell. Clin. Pharm., 14 (1980) 605-608.

Schwartz, P.A., Rhodes, C.T. and Cooper, J.W., Solubility and ionization characteristics of phenytoin. J. Pharm. Sci., 66 (1977) 994-997.

Serajuddin, A.T.M., Factors Influencing the release of phenytoin sodium from slow-release dosage forms. Pharm. Res. 7 (1990) S90.
Sohár, P., NH stretching vibration bands at wave numbers lower than $3000 \mathrm{~cm}^{-1}$ : VI. Cyclic dimeric structures, VI. Hydantoin derivatives. Acta Chim. Acad. Sci. Hung. Tommus, 57 (1968) 425-444.

Weeks, J.D. and Gilmer, G.H., Dynamics of crystal growth. Adv. Chem. Phys., 40 (1979) 157-228. 